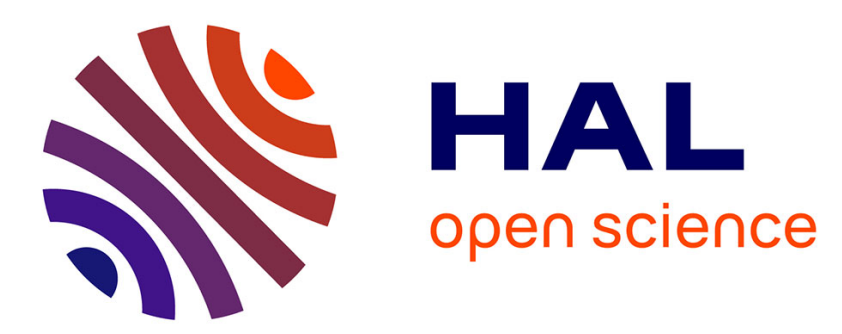

\title{
Determination of small soot particles in the presence of large ones from time-resolved laser-induced incandescence
}

\author{
E Cenker, G Bruneaux, T Dreier, C Schulz
}

\section{- To cite this version:}

E Cenker, G Bruneaux, T Dreier, C Schulz. Determination of small soot particles in the presence of large ones from time-resolved laser-induced incandescence. Applied Physics B Photophysics and Laser Chemistry, 2015, 118 (2), pp.169-183. 10.1007/s00340-014-5966-z · hal-01121150

\author{
HAL Id: hal-01121150 \\ https://hal.science/hal-01121150
}

Submitted on 27 Feb 2015

HAL is a multi-disciplinary open access archive for the deposit and dissemination of scientific research documents, whether they are published or not. The documents may come from teaching and research institutions in France or abroad, or from public or private research centers.
L'archive ouverte pluridisciplinaire HAL, est destinée au dépôt et à la diffusion de documents scientifiques de niveau recherche, publiés ou non, émanant des établissements d'enseignement et de recherche français ou étrangers, des laboratoires publics ou privés. 


\title{
Determination of small soot particles in the presence of large ones from time-resolved laser-induced incandescence
}

\author{
E. Cenker ${ }^{1,2,3}$, G. Bruneaux ${ }^{1,2}$, T. $\operatorname{Dreier}^{3}(\bowtie)$, and C. Schulz ${ }^{3}$ \\ ${ }^{1}$ IFP Energies Nouvelles, 1 et 4 avenue de Bois-Préau, 92852 Rueil-Malmaison, France \\ 2 École Centrale Paris, Paris, France \\ ${ }^{3}$ Institute for Combustion and Gas Dynamics - Reactive Fluids (IVG) and Center for Nanointegration \\ Duisburg-Essen (CENIDE), University of Duisburg-Essen, Duisburg, Germany \\ emre.cenker@uni-due.de \\ gilles.bruneaux@ifpen.fr \\ $\triangle$ thomas.dreier@uni-due.de (corresponding author) \\ christof.schulz@uni-due.de
}

\begin{abstract}
Information about the polydispersity of soot can in principle be gained from time-resolved laser-induced incandescence (LII) using pre-assumed particle-size distributions. This paper introduces an alternative method, called two-exponential reverse fitting (TERF) that is based on combined mono-exponential fits to the LII signal decay at various delay times. The method approximates the particle-size distribution as a combination of one large and one small monodisperse equivalent mean particle size and does not require a distribution assumption. It also provides a ratio of the contribution of both size classes. The systematic error caused by describing LII signals by mono-exponential decays is calculated as less than 2\% for LII signals simulated for monodisperse aggregated soot with heat-up temperatures for which evaporation is negligible. The effects of particle size, heat-up temperature, aggregate size, and pressure on this error are evaluated. The method is tested on simulated LII signals for lognormal and bimodal size distributions and applied to LII data acquired in a laminar non-premixed ethylene/air flame at various heights above burner. The results are compared to transmission electron microscopy (TEM) measurements of thermophoretically-sampled soot. The particle size of the large particle-size class evaluated with the method showed good consistency with TEM results, however the size of the small particle-size class and the relative contribution could not be compared due to missing information in the TEM results for small particles. These limitations of TEM measurements are discussed and the effect of the exposure time of the sampling grid is evaluated.
\end{abstract}




\section{Introduction}

Emission of particulate matter (PM) in combustion processes is a major issue due to its negative impacts on human health and world climate. It is therefore of great interest to understand soot formation processes well enough that new concepts can be developed that help limit these emissions. Powerful diagnostics tools are required that measure the properties of soot in various environments. The primary soot particle diameter $d_{\mathrm{p}}$ is one key indicator for interpreting the effects of soot formation and burnout. Soot usually has polydisperse size distributions and small and large particles show a distinctly different behavior in terms of transport, reactivity, and potential health effects. Information about the size distribution is therefore important for a better understanding of soot formation and oxidation.

Time-resolved laser-induced incandescence (TiRe-LII) is an optical in situ technique for measuring the particle size [1]. Soot particles are heated via absorption of light from a laser pulse to temperatures above $3000 \mathrm{~K}$ and the subsequent blackbody radiation is recorded during the cooling phase. Small particles cool down faster than large ones due to their larger surface-to-volume ratio and therefore provide signal with shorter decay times. Quantitative particle-size information can be obtained from a best-fit comparison of the temporal signal decay and simulations based on the particle's energy- and mass-balance equations [2-4].

Regardless of the actual particle size distribution, a direct fitting for the entire signal trace yields a monodisperse equivalent mean particle size that is known to be biased towards larger sizes for polydisperse ensembles [5]. By assuming a size distribution, information about the different particle-size groups (the shape of a size distribution and its mean value) can be gained from TiRe-LII. Lognormal size distributions are good approximations for various flame conditions and are widely assumed for data evaluation [2,6-8]. Nevertheless, it is generally challenging to fit polydisperse distributions to LII signaldecays because the fitting problem is ill-posed [9]. Particle-size distributions with various combinations of count median diameter $d_{\mathrm{cmd}}$ and geometric standard deviation $\sigma_{\mathrm{g}}$ might produce identical simulated LII signal-decay profiles and therefore the evaluation of measured data does not always lead to unique solutions. Also, a lognormal size distribution may not always be the best assumption. It is reported that multi-lognormal [10] or bimodal distributions [11,12] may show better agreement for some specific environments. Dankers et al. [13] showed a rather simple engineering approach that determines $d_{\mathrm{cmd}}$ and $\sigma_{\mathrm{g}}$ from exponential fits to the LII signal decay at two different delay times and within 
predetermined time intervals. Nevertheless, this method is also based on pre-assumed lognormal distributions.

In this paper a new strategy is presented for determining particle-size information from TiRe-LII. We call this signal evaluation concept TERF (Two-Exponential Reverse Fitting). The method shows some similarity to Dankers et al. [13] because it also uses a simple exponential fit for a delayed part of the LIIsignal trace but in contrast it does not require to pre-assume a particle-size distribution. The TERF method differentiates between signal contributions from small and large particles. It determines monodisperse equivalent mean particle sizes for both size groups and evaluates the relative ratio of particle number densities for both groups.

The evaluation is based on a simplifying exponential approximation. To verify its applicability its variation from a simulated ideal LII-signal trace is evaluated by using least-squares fitting. The influence of various experimental conditions on this approximation, such as heat-up temperature, pressure, particle size, and aggregate size is quantitatively investigated. The novel particle-sizing method is tested on phantom signals that were simulated for well-defined conditions and particle-size distributions. Finally, the method is applied to experimental LII data acquired in a co-annular laminar non-premixed ethylene/air flame at various heights above the burner (HAB). The results are compared to transmission electron microscopy (TEM) measurements of thermophoretically-sampled soot at the same locations.

\section{Theory}

The methodology for TiRe-LII particle-sizing is described, e.g., in [3]. In the present work we use the original code for LIISim [4] imbedded in Matlab routines and details of all routines in this tool are presented, e.g., in [7]. For atmospheric conditions, considerable evaporation starts at around $3400 \mathrm{~K} \mathrm{[6]}$ and becomes stronger with increasing temperature. The present modeling and experimental study is aimed at avoiding these complications by keeping laser fluences below levels where significant evaporation takes place, i.e., keeping the maximum heat-up temperature below the vaporization threshold. In all simulations, the particles were assumed to be graphite-like using the respective materials properties [11]. For heat conduction the Fuchs approach [14] is chosen in LIISim that is known as the most appropriate model for particle-gas energy exchange in a wide range of gas pressures [15]. 


\subsection{Polydispersity}

The TiRe-LII signal acquired from polydisperse soot is the superposition of individual decaying functions for the various size classes weighted by their respective probability $d f$. For a lognormal distribution, $d f$ of a particle size class between $d_{\mathrm{p}}$ and $d_{\mathrm{p}}+\mathrm{d} d_{\mathrm{p}}$ is

$d f=\frac{1}{\sqrt{2 \pi} d_{\mathrm{p}} \ln \sigma_{\mathrm{g}}} \exp \left[-\frac{\left(\ln d_{\mathrm{p}}-\ln d_{\mathrm{cmd}}\right)^{2}}{2\left(\ln \sigma_{\mathrm{g}}\right)^{2}}\right] d \mathrm{~d}_{\mathrm{p}}$

Evaluating the particle size from such a signal with a monodisperse assumption yields a monodisperse equivalent mean particle size that is biased towards larger sizes. The magnitude of this bias is related to the width (i.e., $\sigma_{\mathrm{g}}$ ) of the distribution. To quantify the bias, a set of LII signals was simulated with a polydisperse (lognormal) particle ensembles for $d_{\text {cmd }}$ varying from 10 to $50 \mathrm{~nm}$ in $1 \mathrm{~nm}$ increments and $\sigma_{\mathrm{g}}$ varying from 1.1 to 1.8 in 0.02 increments. To ensure that each signal trace covers the entire process from reaching the peak temperature back to thermal equilibrium with the bath-gas, the duration of each simulation was set to $2 \mu \mathrm{s}$. Input parameters are given in Tab. 1 . For each case, the signal was then evaluated using a monodisperse approach.

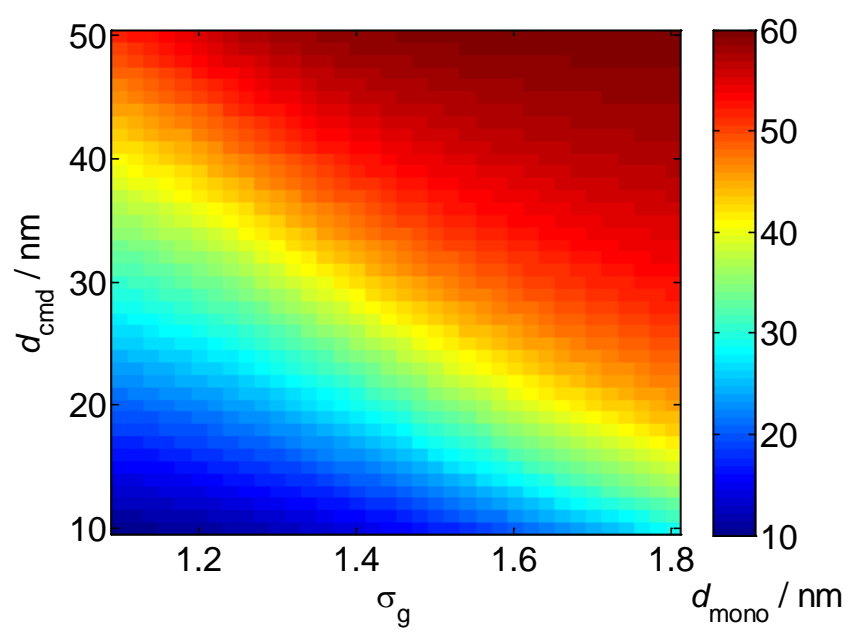

Fig. 1: Monodisperse particle sizes evaluated from synthetic LII signals simulated for lognormal particle-size distributions with various values of $d_{\mathrm{cmd}}$ and $\sigma_{\mathrm{g}}$.

The results for the monodisperse response to the polydisperse input are shown in Fig. 1 . The determination of the particle-size distribution using an inverse approach is an ill-posed problem. To better illustrate this, six TiRe-LII signal traces with the same monodisperse equivalent mean particle size of $40.2 \mathrm{~nm}$ (same color code in Fig. 1) but differing values of $d_{\mathrm{cmd}}$ and $\sigma_{\mathrm{g}}$ are selected. The almost 
identical LII-signal traces are plotted on Fig. 2a. Fig. $2 \mathrm{~b}$ shows the strongly varying underlying particlesize distributions with the respective color code (each data point on the distribution curve represents the probability density of a $1 \mathrm{~nm}$-wide bin).
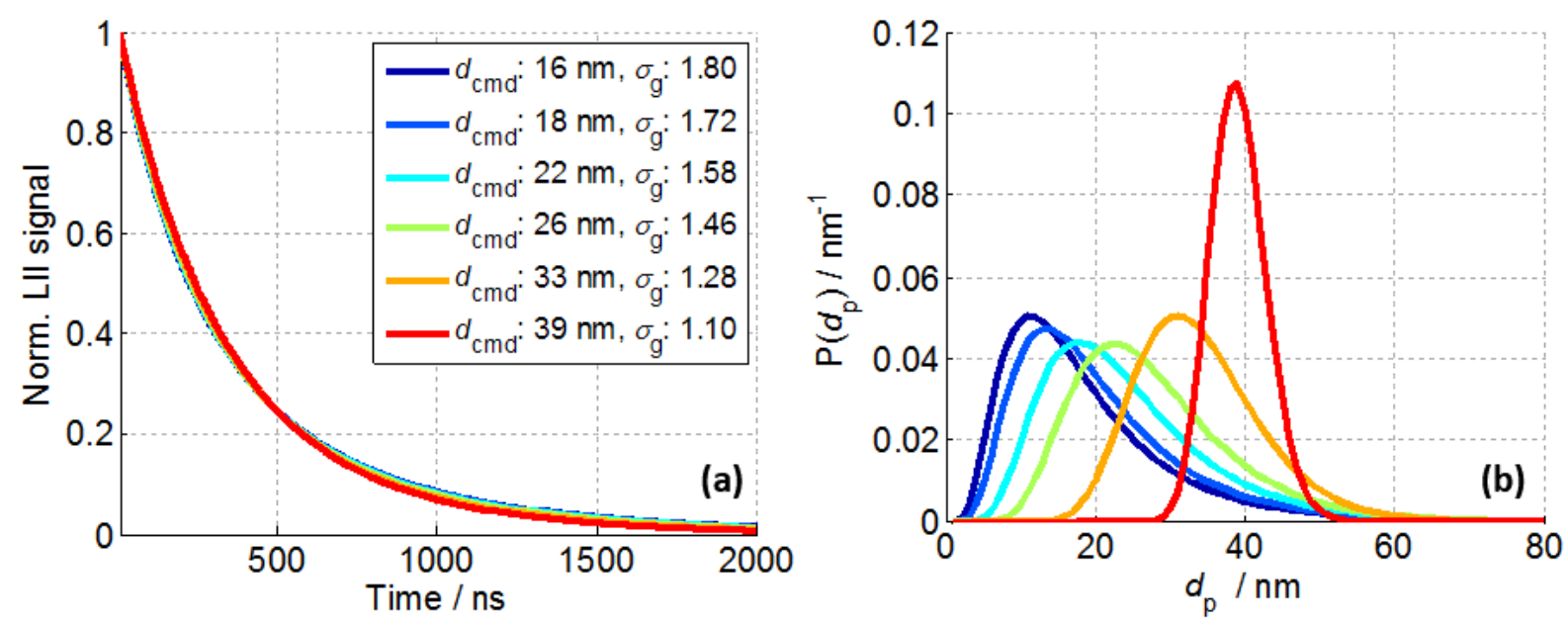

Fig. 2: (a) TiRe-LIl signals simulated for soot ensembles with the particle size distributions shown in (b).

This comparison makes clear that signal interpretation based on standard inversion schemes suffers from this ambiguity with a fairly wide range of satisfactory solutions. To overcome this problem, various approaches were proposed and Daun et al. [16] reviewed various implicit solution schemes for recovering particle-size distributions from TiRe-LII data. It is shown that such implicit solution approaches suffer loss of accuracy when the input data is slightly modified or when the noise contribution to the measured signal is considerable. Furthermore, approaches that aim at solving the forward problem are time intensive, thus they are not suitable for on-line analysis.

\subsection{Simple exponential approximation}

In the absence of strong evaporation, heat conduction is the dominant cooling mechanism of soot particles after laser heating. During this cooling, the decay rate remains almost unchanged for an isolated particle or a soot aggregate composed from monodisperse particles, and therefore the LII signal can be approximated by a simple exponential decay [17]:

$y=k e^{-t / \tau}$

Where $k$ is the pre-exponential factor, $t$ is the time and $\tau$ is the lifetime of the LII signal of the respective size group. To verify the accuracy of this approximation, a "control LII signal" is simulated for the input 
parameters shown in Tab. 1 that is later on used as a reference case for comparison with simulated signals with varying input parameters.

Tab. 1: Input parameters for modeling the standard "control signal".

\begin{tabular}{|l|c|}
\hline Property & Standard condition \\
\hline Particle-size distribution & monodisperse \\
\hline Particle size & $30 \mathrm{~nm}$ \\
\hline Aggregate size & $1 \mathrm{bar}$ \\
\hline Ambient pressure & $1750 \mathrm{~K}$ \\
\hline Bath-gas temperature & $3300 \mathrm{~K}$ \\
\hline $\begin{array}{l}\text { Maximum heat-up } \\
\text { temperature }\end{array}$ & 0.25 \\
\hline $\begin{array}{l}\text { Thermal accommodation } \\
\text { coefficient }\end{array}$ & 0.4 \\
\hline E(m) & $410-440 \mathrm{~nm}$ \\
\hline Detection spectral range & \\
\hline
\end{tabular}

An exponential function is fitted to this control signal with a least-squares minimization routine for the time range where the LII signal intensity decays to $5 \%$ of its peak value. Both curves are shown in Fig. 3 .

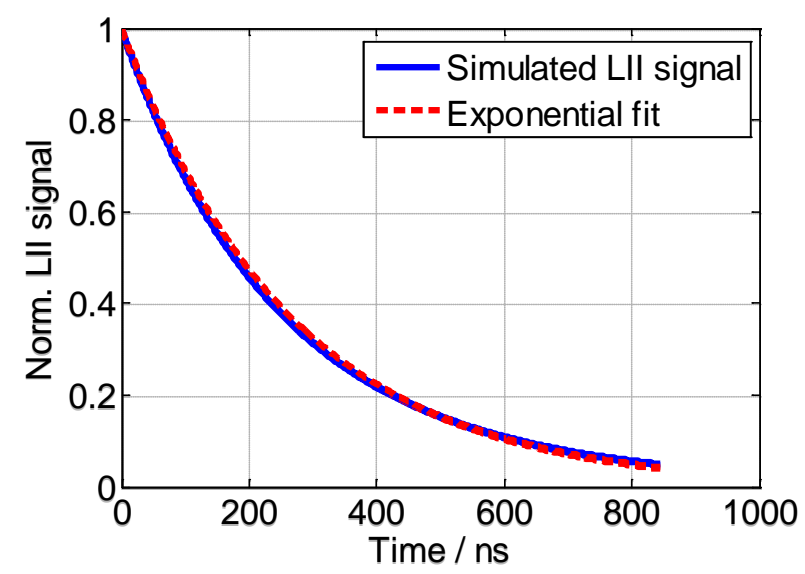

Fig. 3: Simulated LII signal for a monodisperse aggregate (conditions given in Tab. 1) and a mono-exponential fit.

The goodness of the fit $R^{2}$ is over 0.9994. To quantify the systematic error in particle sizing due to such an approximation, LII-signal curve-fitting is performed by using the new exponential fit (red dashed curve) and the identical input parameters of the control LII signal as model input. This fitting yielded a particle size of $30.6 \mathrm{~nm}$ which is only $2 \%$ larger than the original input. This minor deviation results from the small contributions of radiation and evaporation to the total cooling. Each of these heat exchange mechanisms contributes its own independent exponential decay rate leading to a deviation from the 
mono-exponential decay. Because of the low heat-up temperature in the control case, the difference, however, is negligible.

For the validity of the mono-exponential approximation, the experimental conditions (i.e., input parameters of the simulation) are important as they influence the relative importance of the various heat-exchange mechanisms. To quantify this influence, the same procedure is repeated by systematically changing one individual input parameter (keeping all others fixed), and the resulting systematic errors (i.e., the difference caused by the exponential approximation relative to the input data) are shown in Fig. 4 for varying pressure, particle size, aggregate size, and heat-up temperature. Unless otherwise stated, each simulation is performed with the conditions shown in Tab. 1.
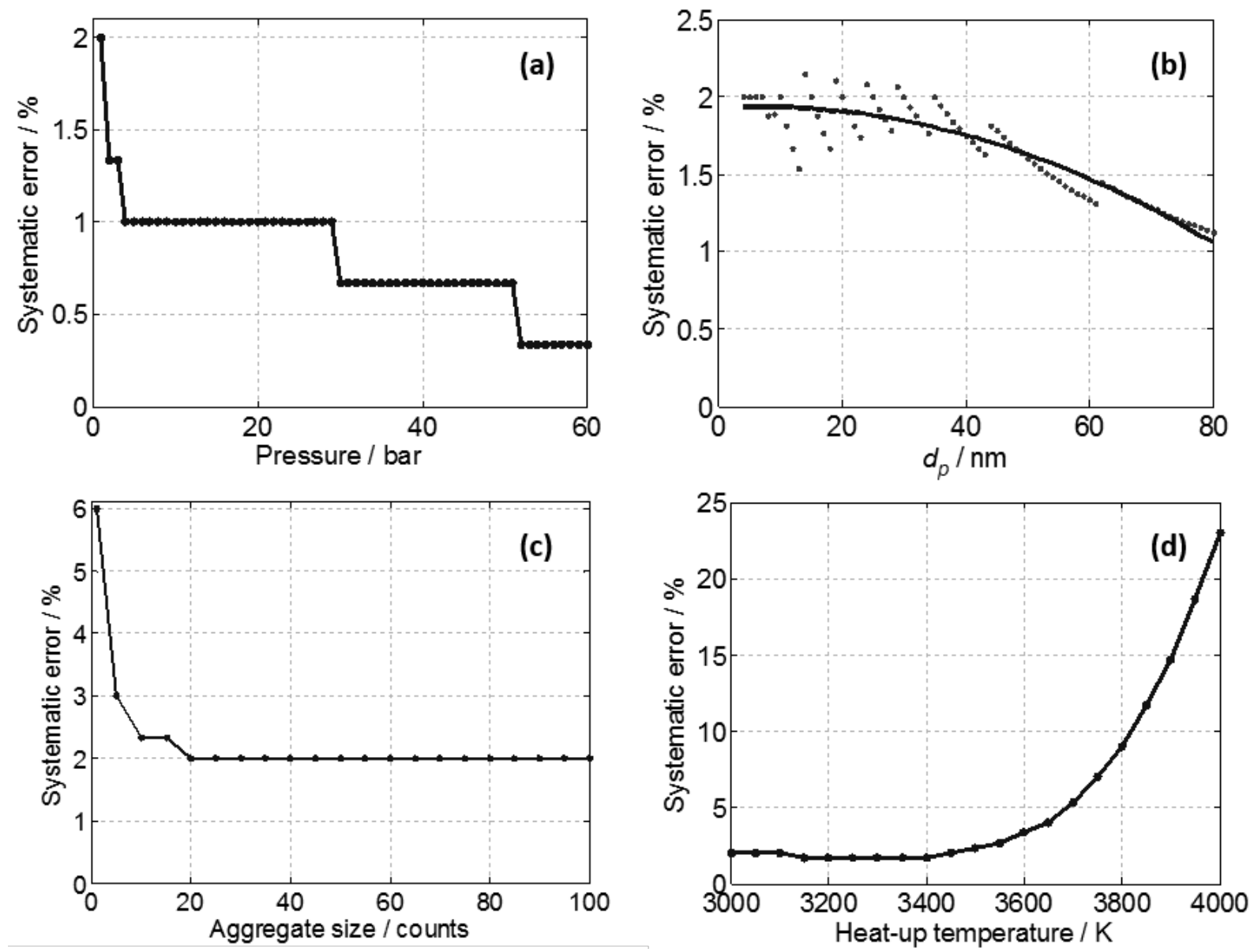

Fig. 4: Systematic errors in particle-sizing due to approximating a simulated LII decay with a mono-exponential decay for varying pressure (a), particle size (b), aggregate size (c), and heat-up temperature (d).

In many practical combustion devices soot is formed at elevated pressure. At high pressure, the LIIsignal lifetime is significantly reduced due to enhanced heat transfer caused by increased collision rates. 
In comparison, pressure has a negligible effect on the evaporation rate and radiation is not affected at all. Therefore, with increasing pressure, the importance of conduction in the total heat exchange further increases and the LII signal converges to a simple exponential decay. Fig. 4a shows this effect for pressures from 1 to 60 bar in 1 bar increments. The systematic error further reduces from $2 \%$ at 1 bar to below $0.5 \%$ at high pressures. The particle-sizing routine of LIISim has a resolution of $0.1 \mathrm{~nm}$. Therefore, the evaluated systematic error shows distinct steps as a function of pressure.

The systematic error evaluated for particles from 4 to $80 \mathrm{~nm}$ with $1 \mathrm{~nm}$ increments is shown in Fig. $4 \mathrm{~b}$ (4 $\mathrm{nm}$ is the minimum input value for LIISim). The actual data is shown with black dots and the curve is a second-order polynomial fit to illustrate the trend. The oscillation of the data is caused by the $0.1 \mathrm{~nm}$ resolution of LIISim. Theoretically, No dependence on the error on particle size is expected because all the heat exchange mechanisms are affected equally. Nevertheless, a slight $1 \%$ change in the systematic error is observed over the given particle size range (systematic error starts to reduce after around 40 $\mathrm{nm})$. This is, however, a result of the data format of LIISim. By default, LIISim uses the first microsecond of the data only for fitting. When the particle size increases from 4 to $80 \mathrm{~nm}$, the LII-signal lifetime increases from $\sim 30 \mathrm{~ns}$ to $\sim 2 \mu \mathrm{s}$ (it exceeds $1 \mu \mathrm{s}$ at around $40 \mathrm{~nm}$ ). With large particles, a part of the signal trace is thus disregarded. Such a partial using of the LII signal results in a better fitting by a simple exponential function. Therefore, for particles larger than $40 \mathrm{~nm}$ smaller systematic errors are observed. If the curve-fitting routine was not limited in time, no particle-diameter dependence would occur.

Soot aggregates can be described as random fractal structures. In LII modeling, the evaporation rate, the change in internal energy and the heat loss due to radiation are not affected by aggregation as long as the individual particles remain in the Rayleigh regime. The signal scales linearly with the number of primary particles within an aggregate and hence the mass [18]. However, heat conduction is affected by aggregation. A primary soot particle within an aggregate cools down more slowly than an isolated one because of shielding by the surrounding particles [19]. Fig. 4c shows the systematic error for an isolated particle and aggregates with 5 to 100 particles. With increasing aggregate size, the contribution of heat conduction to the total cooling decreases which leads to the assumption of an increasing deviation from a mono-exponential decay. Surprisingly, the results show the opposite trend. The greatest change occurs when switching from isolated particles to the aggregate model. For aggregates larger than 20 particles the magnitude of the change is below the resolution of LIISim. This contradiction requires further investigation and might indicate an incorrect treatment of shielding effects in the underlying submodel in LIISim. 
The particle heat-up temperature reached during the laser pulse depends on laser fluence. A change of this parameter influences the heat exchange rates of radiation, conduction, and evaporation. The most critical change occurs with evaporation when the temperature exceeds approx. $3400 \mathrm{~K}$ (Fig. 4d). Its contribution to the total cooling increases significantly which causes a strong deviation from the simple exponential decay.

All these analyses indicate that, compared to other uncertainties in LII particle-sizing [20], the error due to the mono-exponential approximation is minor for monodisperse particle ensembles heated in the low-fluence regime. Deviating from the low-fluence regime, however, causes a strong deviation from the mono-exponential assumption. Increasing pressure in contrast is beneficial. Hence, to benefit from the simplicity of fitting exponential functions, this approximation can be used as a tool for further detailed size-distribution analysis.

\subsection{Size-distribution analysis with the TERF method}

In polydisperse particle ensembles where each size group has a unique LII-signal lifetime, the signal superposition causes a deviation from the mono-exponential decay behavior. The relative contribution of each size group is linearly proportional to its relative volume fraction $f_{v}$. Hence, such a LII signal cannot be accurately approximated by mono-exponential decay. Nevertheless, with increasing time after the laser heat-up, the particle temperature depends on size. Because smaller particles cool faster than larger ones, their contribution to the overall LII signal decreases over time and the remaining signal becomes increasingly dominated by the larger particles [2].

The TERF method couples the fact that the LII signal is dominated by large particles at long delay times and the mono-exponential approximation to derive information about the particle-size distribution. When starting the signal evaluation after a certain delay after laser heat-up, the remaining signal trace preferentially represents the largest particles. Although this signal still contains contributions from particles with various particle sizes, the relative size distribution is narrowing with increasing delay. For such narrow distributions, it has been shown $[9,13]$ that particle-sizing via a monodisperse fitting yields a good approximation for the mean particle size. Therefore, mono-exponential fitting eventually becomes possible with an acceptable uncertainty that represents the large particles in the ensemble. Once the parameters of the mono-exponential fit are determined, the respective signal contribution of the subsection of the particle ensemble can be extrapolated back in time into the time domain before the above-mentioned delay and into the data disregarded before. This extrapolated curve represents 
the theoretical cooling of the large particle fraction right after the laser pulse and has a slower decay rate than the overall signal. The additional signal (the difference between the measured signal and the back-extrapolated signal) then stems from smaller particles.

To illustrate this approach, a LII-signal trace is simulated with a wide lognormal distribution $\left(d_{\mathrm{cmd}}=20\right.$ $\mathrm{nm}, \sigma_{\mathrm{g}}=1.8$, black curve in Fig. 5a). All the other parameters are those from Tab. 1 . The delay time for starting the data evaluation is $300 \mathrm{~ns}$ (dash-dotted grey vertical line) and a mono-exponential function is fitted to the delayed signal portion that is then extrapolated back to the 0-300 ns time range (dashed red line). The original LII signal has a steeper decay than that of the back-extrapolated fit and it is stronger because of the additional contribution of signal from small particles (shown by the blue dotted curve). The comparison of the normalized curves (Fig. 5b) shows the difference in decay times and thus the origin from different sub-sections of the particle-size ensemble. It should be noted that the signal representing the small particle class (blue dotted line) is also composed of contributions from various particle sizes, however the distribution is again narrower than that of the total ensemble.
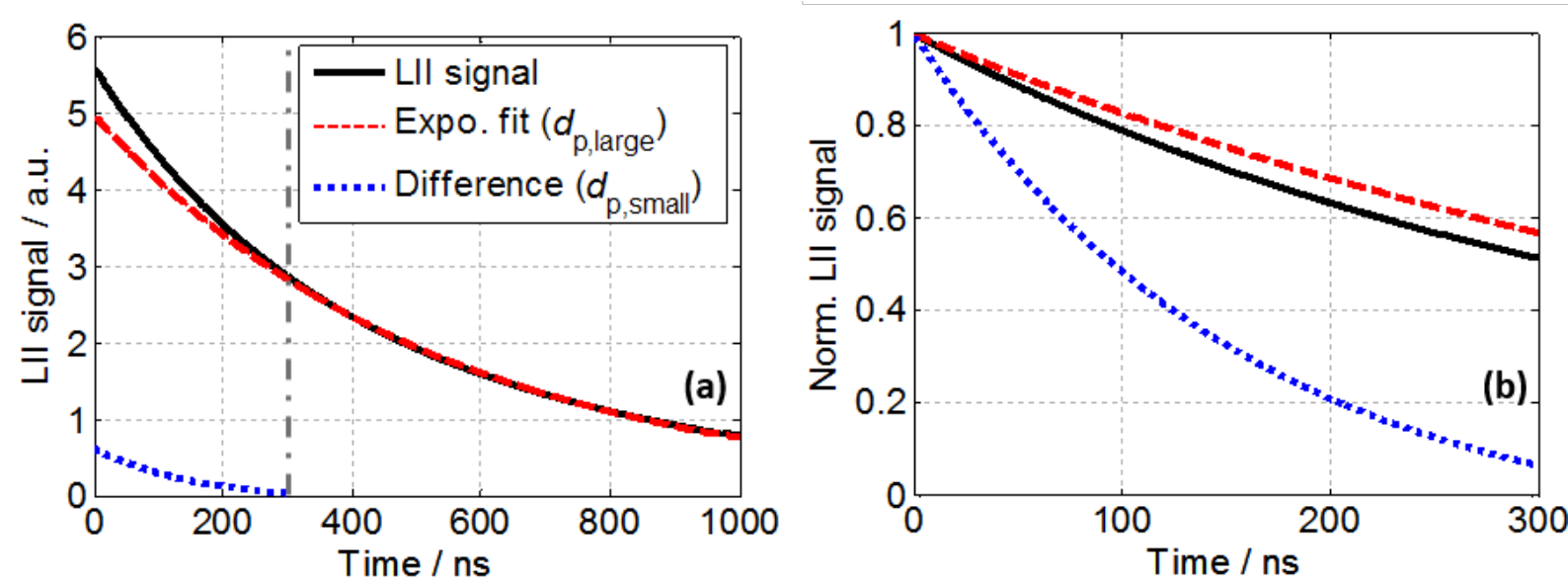

Fig. 5: (a) Simulated LII signal for polydisperse soot with a lognormal distribution $\left(d_{\mathrm{cmd}}=20 \mathrm{~nm}, \sigma_{g}=1.8\right.$, black solid line). A mono-exponential decay is fitted to the section of the LII signal from $300 \mathrm{~ns}$ down to $5 \%$ of the peak signal and extrapolated back into the time domain from 0 to 300 ns (red dashed line). The difference between both curves represents the signal contribution from small particles (blue dotted line). (b) The same data normalized to the respective peak values.

A monodisperse equivalent particle size can be evaluated from each of these LII signals representing small and large particles, respectively. The evaluation of the time-delayed data requires the knowledge of the actual temperature of the particles at that delay as heat-up temperature for the LIISim simulation. When calculating the extrapolated exponential curve starting at $0 \mathrm{~ns}$, the original heat-up temperature 
can be used and a temporally-resolved temperature information is not necessary. In Fig. 6, the evaluated small and large monodisperse equivalent particle sizes, $d_{p, \text { small }}=15 \mathrm{~nm}$ and $d_{\mathrm{p}, \text { large }}=57 \mathrm{~nm}$, are shown along with the actual distribution of the particle size used for the simulation (each data point on the distribution curve represents the probability density of a 1-nm wide bin). These particle sizes represent a mean of different size classes at the two ends of the distribution curve. When particle sizing is performed with the entire LII signal (from peak signal to $5 \%$ of this peak) using a monodisperse approach, the evaluated particle size is $45.3 \mathrm{~nm}$.

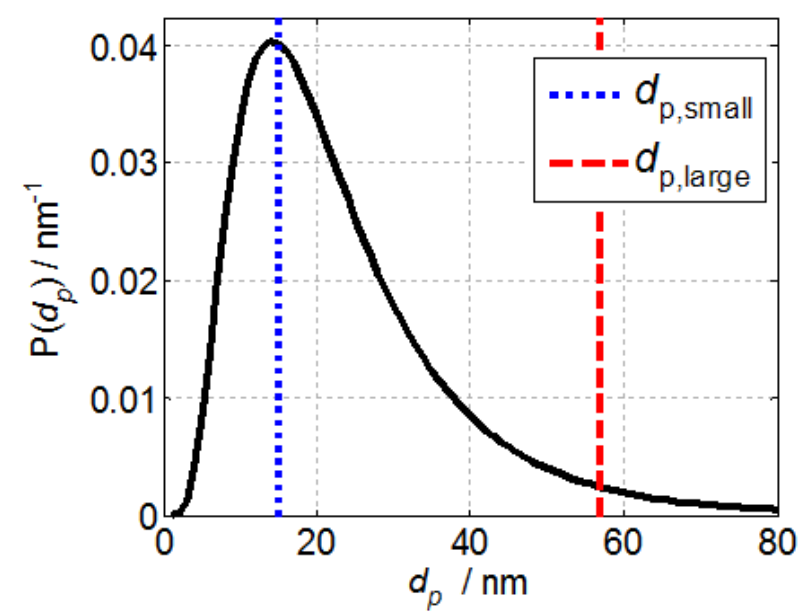

Fig. 6: Lognormal distribution function of particle sizes for $d_{\mathrm{cmd}}=20 \mathrm{~nm}$ and $\sigma_{\mathrm{g}}=1.8$. The vertical lines show the monodisperse equivalent particle sizes evaluated from the exponential decays (see text) representing the small and large particle class of $d_{\mathrm{p}, \text { small }}=15 \mathrm{~nm}$ and $d_{\mathrm{p}, \text { large }}=57 \mathrm{~nm}$, respectively.

In addition to the mean particle sizes from both size classes, the ratio of their number densities can be evaluated from the pre-exponential intensities with the TERF method. In a monodisperse soot ensemble, the LII signal is linearly proportional to the soot volume fraction [21] which can be defined in terms of the primary particle diameter, and the average number of primary particles, $n_{\mathrm{p}}$ :

$f_{\mathrm{v}}=\frac{\pi}{6} n_{\mathrm{p}} d_{\mathrm{p}}^{3}$

The magnitude of the LII signal at $t=0$ for the small and large particle-size classes where all particles are at the same temperature (at least for atmospheric or sub-atmospheric pressure, see [5]), is given in Fig. 5 a by the ratio of the LII signal intensities, $S_{\text {LII }}(0)$ at the time of the laser pulse $(t=0 \mathrm{~s})$ : 
$\frac{S_{\text {LII,small }}}{S_{\text {LII,large }}}=\frac{n_{\mathrm{p}, \text { small }}}{n_{\mathrm{p}, \text { large }}} \frac{d_{\mathrm{p}, \text { small }}^{3}}{d_{\mathrm{p}, \text { large }}^{3}}$

The ratio of number densities, $n_{\mathrm{p}, \text { small }} / n_{\mathrm{p} \text {,large }}$ (denoted with $\mathcal{R}_{n}$ hereafter) is calculated as 12.9 . The actual ratio for the identical $d_{\mathrm{p}, \text { small }}$ and $d_{\mathrm{p} \text {, large }}$ is 14.4 over the assumed lognormal distribution curve.

Because the particle-size distribution is a continuous function, the diminishing contribution of the small particles to the overall signal also continuously decreases with time. Therefore, there is not a perfect delay time that can be defined to separate the range of small and large particles. To see the effect of various chosen delay times, this value is changed from 10 to $600 \mathrm{~ns}$ in $10 \mathrm{~ns}$ increments while determining $d_{\mathrm{p}, \text { small }}, d_{\mathrm{p}, \text { large, }}$ and $\mathcal{R}_{n}$ (Fig. 7).
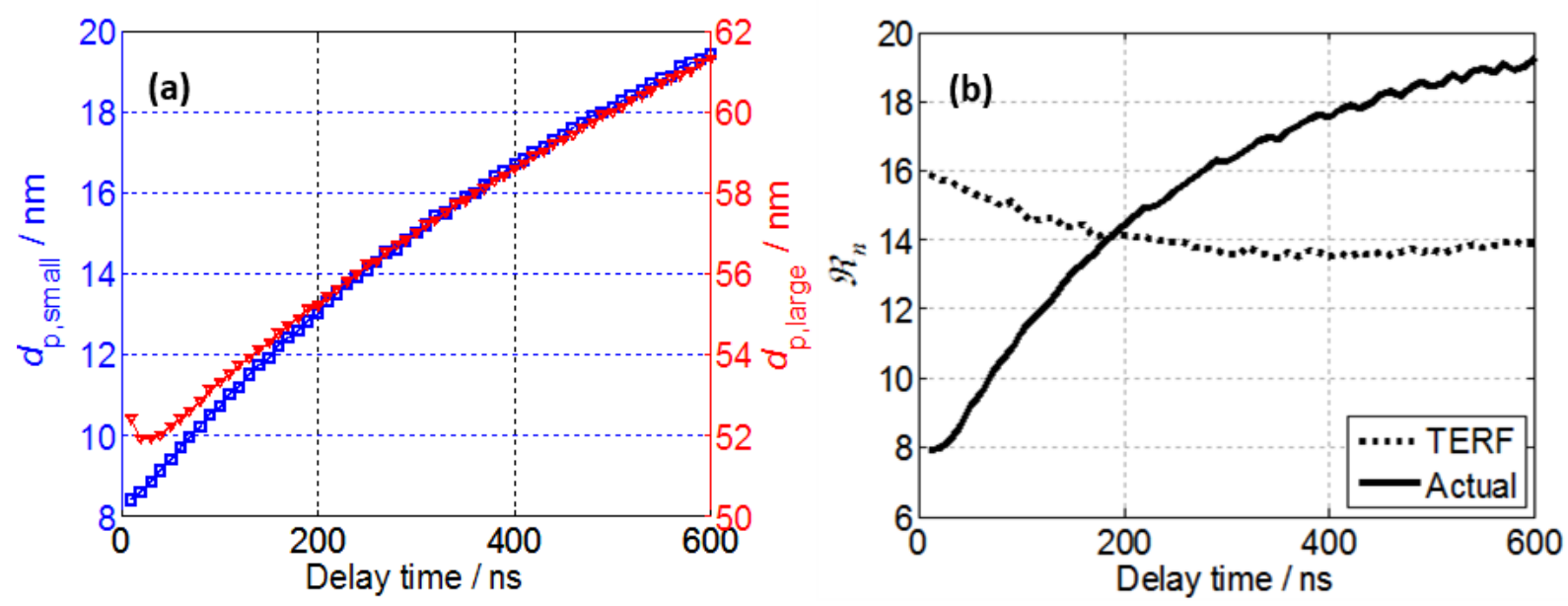

Fig. 7: (a) $d_{\mathrm{p}, \text { small }}$ and $d_{\mathrm{p}, \text { large }}$ and (b) ratio of the number densities as a function of the chosen delay time.

As the delay time increases, the contribution of the small particles to the signal segment after the delay gradually decreases, and the lifetime of this signal segment increases resulting in evaluation of larger particles. Similarly, when the delay time increases, the difference curve representing the cooling of the small particles contains a larger contribution from larger particle classes, and consequently the evaluated particle size increases (Fig. 7a). However, an optimum delay time range exists for this strategy. When the delay time is too short, the latter segment used for the exponential fit contains too much information from the different size classes leading to a severe reduction of the fitting accuracy. Likewise, with a too large delay, the weight of larger particles in the difference curve increases and the evaluated $d_{p, \text { small }}$ does not represent an accurate mean size for the smallest size classes. The $\mathcal{R}_{n}$ curves in Fig. 7b also verify this argument: For too early or too late delay times, the predicted results with the TERF method deviate from the actual $\mathcal{R}_{n}$ that is evaluated from the original distribution. For the present 
conditions, a delay time at around 200 ns gives a ratio close to the actual distribution. This optimum delay may vary for different distributions (this will be further analyzed in section 4.2).

\subsection{Evaluation of various particle-size distributions}

The strategy described before was tested on polydisperse soot ensembles with various distribution characteristics. In addition to the wide lognormal distribution introduced in section 2.3, a narrower lognormal distribution and two bimodal distributions with distinctive shapes were analyzed. The bimodal distribution functions, $p_{\text {bimodal, }}$ were created from two standard lognormal distributions superimposed with independent weight functions [11]:

$p_{\text {bimodal }}=w_{1} p_{1}\left(d_{\mathrm{cmd}, 1}, \sigma_{\mathrm{g}, 1}\right)+w_{2} p_{2}\left(d_{\mathrm{cmd}, 2}, \sigma_{\mathrm{g}, 2}\right)$

$p_{1}$ and $p_{2}$ are the probability density functions with lognormal distributions, whereas $w_{1}$ and $w_{2}$ are the weight factors for the small-size mode and the large-size mode, respectively. The distribution parameters used in this section are listed in Tab. 2.

Tab. 2: Parameters of the investigated particle-size distributions.

\begin{tabular}{|c|c|c|c|}
\hline Parameters & a & b & c \\
\hline Distribution & Lognormal & Bimodal & Bimodal \\
\hline$d_{\mathrm{cmd}, 1} / \mathrm{nm}$ & 15 & 15 & 15 \\
\hline$\sigma_{g, 1}$ & 1.5 & 1.4 & 2 \\
\hline$w_{1}$ & 1 & 3 & 1 \\
\hline$d_{\mathrm{cmd}, 2} / \mathrm{nm}$ & - & 40 & 30 \\
\hline$\sigma_{g, 2}$ & - & 1.3 & 1.5 \\
\hline$w_{2}$ & - & 1 & 1 \\
\hline
\end{tabular}

The distribution functions are shown in Fig. 8 (each data point on the distribution curves represents the probability density of a $1 \mathrm{~nm}$-wide bin) along with the evaluated small and large monodisperse equivalent mean particle sizes (vertical lines). For all the three cases, the delay time was $220 \mathrm{~ns}$. The actual $\mathcal{R}_{n}$ calculated from the distribution function (ratio of $P\left(d_{\mathrm{p}}\right)$ at $d_{\mathrm{p}, \text { small }}$ and $d_{\mathrm{p}, \text { large }}$ ) and the $\mathcal{R}_{n}$ calculated with the TERF method are given on the respective plots. 

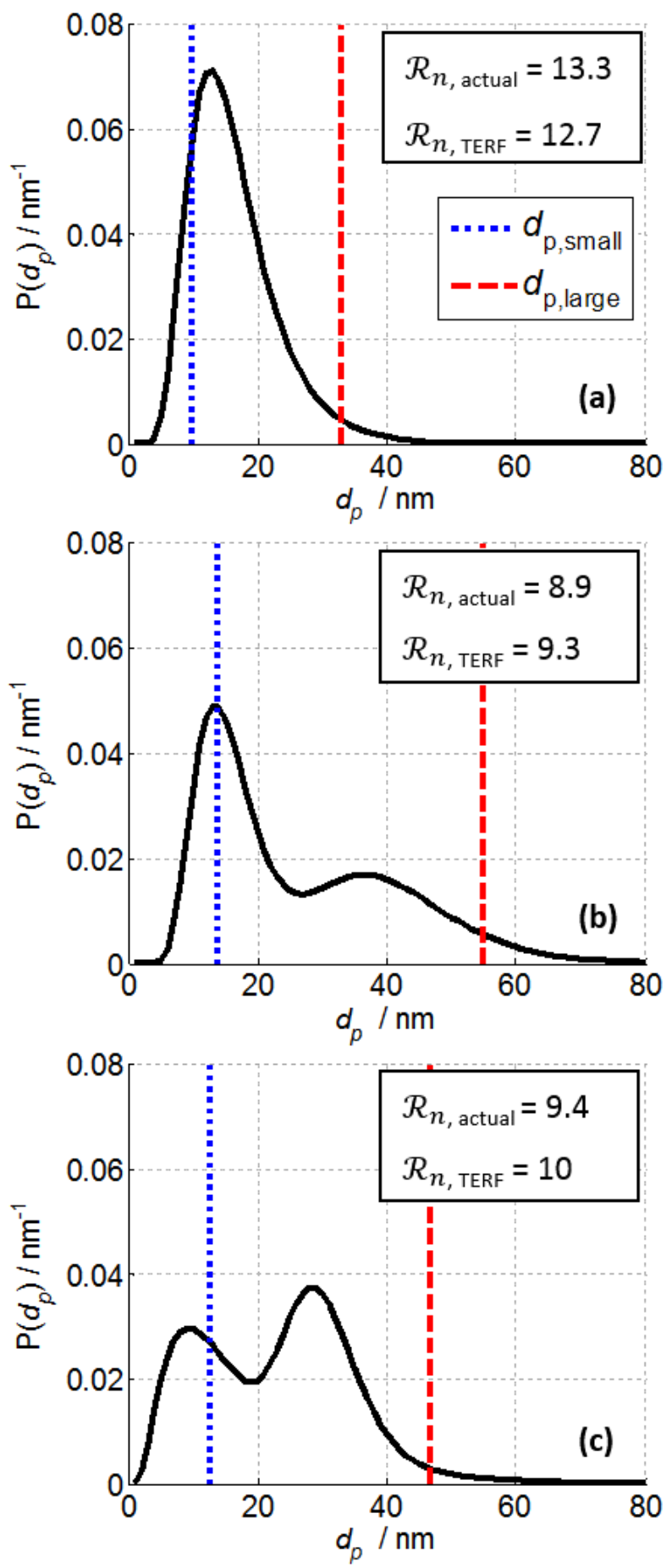

Fig. 8: Probability density functions for lognormal (a) and bimodal (b, c) distributions. Vertical lines show the monodisperse equivalent particle sizes evaluated for the small and large particle classes.

In all three cases the evaluated $d_{p, \text { small }}$ and $d_{p \text {,large }}$ fall within the distribution and represent a mean particle size within their size classes. The $\mathcal{R}_{n}$ values evaluated with the TERF method also shows good agreement with the actual $\mathcal{R}_{n}$ derived from the probability density functions at $d_{\mathrm{p}, \text { small }}$ and $d_{\mathrm{p} \text {,large }}$. 


\section{Experiment}

Particle-size measurements were performed in a non-premixed ethylene/air Santoro flame [22] operated under standard conditions $\left(\mathrm{C}_{2} \mathrm{H}_{4}: 0.232\right.$ standard liters per minute $(\mathrm{s} / \mathrm{m})$, air co-flow: $\left.43 \mathrm{~s} / \mathrm{m}\right)$. To stabilize the flame, a chimney with a $25 \mathrm{~cm}$ diameter was mounted $30 \mathrm{~cm}$ above the burner. The schematics of the experiment is shown in Fig. 9. For LII, the fundamental of a Nd:YAG laser at $1064 \mathrm{~nm}$ was used with a pulse width of $7 \mathrm{~ns}$. A set of cylindrical lenses forms a horizontal laser sheet, and a $1 \mathrm{~mm}$ slit aperture crops the laser sheet into a rectangular shape that is relay-imaged into the center of the flame with a spherical lens at a $2 f$ distance creating a nearly top-hat intensity profile. This spatial profile was measured with a beam profiler. The laser fluence was set to $0.08 \mathrm{~J} / \mathrm{cm}^{2}$ which does not cause any considerable soot evaporation. The LII signal was detected with a fast photomultiplier (PMT, Hamamatsu R7400U-04, rise time $\sim 0.78 \mathrm{~ns}$ ) coupled with a bandpass filter ( $425 \pm 15$ or $676 \pm 14 \mathrm{~nm}$ ) and stored on a $1 \mathrm{GHz}$ oscilloscope. The photomultiplier was combined with a system of two spherical lenses and a circular aperture to provide high spatial resolution. This circular probe zone in the flame has a diameter of $1.5 \mathrm{~mm}$ and it was located at a $2 \mathrm{~mm}$ distance to the burner axis on the pump laser entrance side. This radial location has a higher soot mass with respect to the burner axis location, thus better signal-to-noise ratios can be achieved. The burner and the chimney were mounted on a vertical translation stage independent from the optical path, which enables different heights in the flame to be probed.

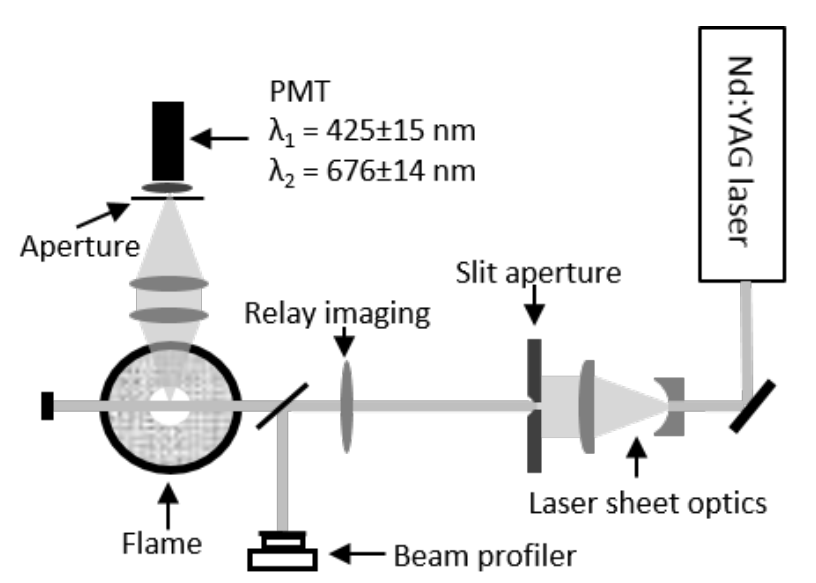

Fig. 9: Experimental arrangement

The measured LII decay curves were recorded at heights between $30-70 \mathrm{~mm}$ above the burner (HAB) in steps of $5 \mathrm{~mm}$. At each location 100 single measurements were recorded with each of the two bandpass 
filters. Prior to the LII measurements, the detection system was spectrally calibrated by replacing the burner with a broad-band halogen lamp with known irradiance.

For ex situ characterization, thermophoretic sampling was achieved at five HAB positions using a pneumatically-driven soot sampling probe on which lacey carbon-coated copper grids were attached. The probe consisted of two thin metallic plates in a sandwich form, with a 3-mm-diameter hole exposing both sides of the TEM grid to the flame (a similar design is shown in ref. [23]). The purpose of using a lacey grid was to minimize the distortion of the electron beam by the carbon film during the microscope imaging. A high-frame-rate camera (1000 fps) positioned perpendicular to the pneumatic cylinder axis was mounted to measure the probe trajectory and the timing precisely. The exposure time of the probe to the flame environment (time duration where the grid rests within the flame) was set to $40 \mathrm{~ms}$ whereas the transit time (where the grid moves within the flame boundary) was shorter than $3 \mathrm{~ms}$. Additional samples were collected with 60 and $80 \mathrm{~ms}$ exposure times at $50 \mathrm{~mm}$ HAB to analyze the effect of exposure time on soot sampling. The radial distance of the grid center was $2 \mathrm{~mm}$ from the burner axis. The sampled soot was analyzed with a Tecnai FEG $200 \mathrm{kV}$ transmission electron microscope and images were recorded at 26000x magnification and micrographs were manually analyzed using the freeware ImageJ. It should be noted that the TEM analysis of grids in this work were conducted by a third party TEM operator who had no extensive experience in soot research. The operator acquired $\sim 40$ images from each grid, and it is lately understood that in each of these images large aggregates were targeted and all the possible isolated particles and small aggregates were omitted. Therefore, the TEM-

derived results in this work do not represent the entire soot ensemble in the respective sampling location.

\section{Results and discussion}

\subsection{Input parameters}

At each probe volume along the flame, the ambient conditions must be known for accurately modeling the LII heating and cooling processes that may vary due to radiative cooling, changing mixture fraction, and changing soot morphology. In this study, the heat-up temperature of the laser-heated soot particles was derived via pyrometry from the ratio of peak LII signal intensities within two detection bands by using a lookup table method [24]. These spectral bands ( $425 \pm 15$ and $676 \pm 14 \mathrm{~nm}$ ) were selected 
according to the recommendation of [25]. To suppress the influence of background signal, i.e., line-ofsight integrated flame luminosity, the baseline signal before the laser pulse was subtracted at each location. To measure the effective bath-gas temperature, the measurements were repeated at $\mathrm{HAB} 60$ $\mathrm{mm}$ without the laser pulse. At around this height the radial temperature gradients are minimal and the temperature is radially uniform [26], thus a biasing towards lower temperatures due to edge effects was negligible. Through subtracting the heat-up temperature from the bath-gas temperature, the temperature increase due to the laser light absorption was calculated as $1650 \mathrm{~K}$ at this height. Assuming a constant soot absorption function $E(m)$ and the same temperature increase at all locations, the bath gas temperatures were calculated for the flame heights. The flame- and heat-up temperatures used for simulating LII signals are shown in Tab. 3.

Tab. 3: Heat-up temperature, bath-gas temperature, and aggregate size input parameters imposed for particlesizing at different heights above burner (HAB).

\begin{tabular}{|c|c|c|c|}
\hline $\mathbf{H A B} / \mathbf{n m}$ & $\boldsymbol{T}_{\text {heat-up }} / \mathbf{K}$ & $\boldsymbol{T}_{\text {gas }} / \mathbf{K}$ & $\boldsymbol{N}_{\mathbf{p}}$ \\
\hline 30 & 3414 & 1764 & 40 \\
\hline 35 & 3390 & 1740 & 50 \\
\hline 40 & 3351 & 1701 & 60 \\
\hline 45 & 3271 & 1621 & 70 \\
\hline 50 & 3259 & 1609 & 80 \\
\hline 55 & 3282 & 1632 & 90 \\
\hline 60 & 3225 & 1575 & 80 \\
\hline 65 & 3235 & 1585 & 70 \\
\hline 70 & 3162 & 1512 & 60 \\
\hline
\end{tabular}

At the investigated radial location bath-gas temperatures decrease with increasing $H A B$ due to the cooling of the flame via radiation and convection (at another radial position different trends might occur depending on air entrainment). Aggregate sizes also changes with flame height [9] and the values shown in Tab. 3 are estimations based on TEM analysis. The other input parameters used at each location are shown in Tab. 1. With a rough estimation, the precision of the heat-up temperature measurement via two-color pyrometry can be taken as $\pm 100 \mathrm{~K}$, the bath gas temperature $\pm 200 \mathrm{~K}$ and the aggregate size \pm 40 particles. The influence of these uncertainties on the evaluated particle size is shown in Fig. 10 . The total uncertainty of the evaluated particle size due to these input parameters is approximately $\pm 20 \%$. 

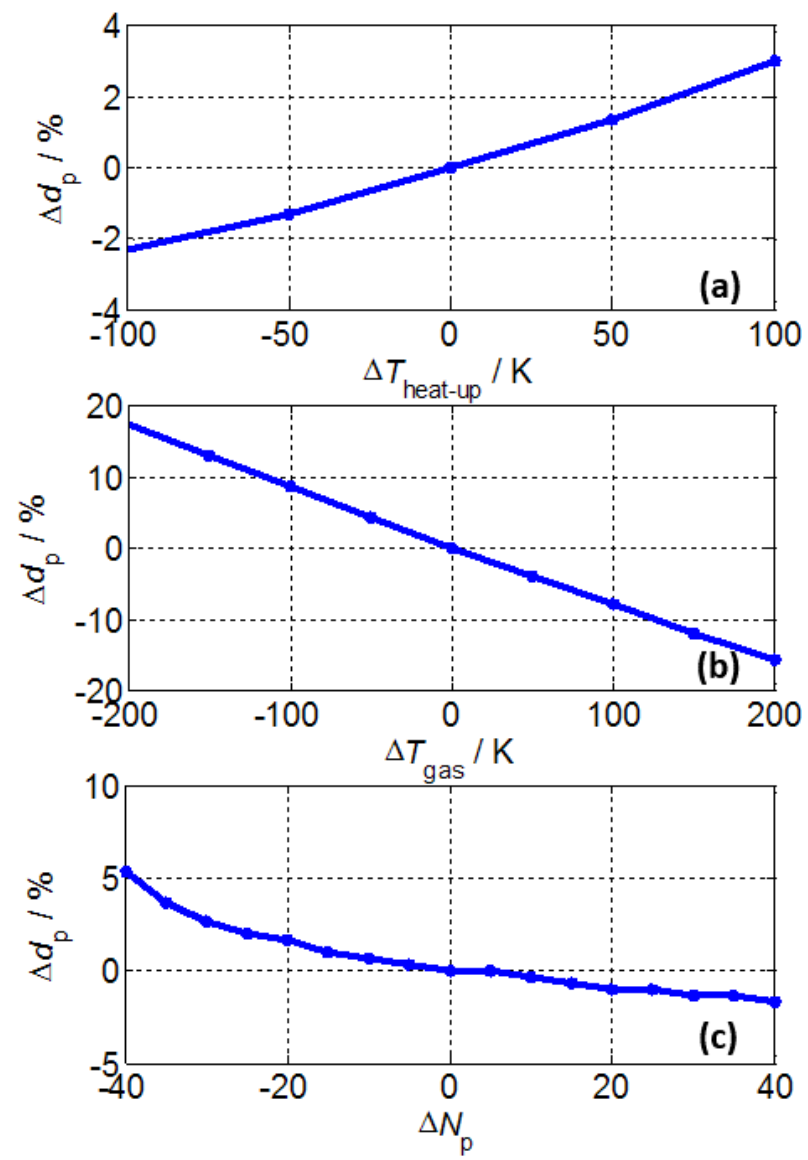

Fig. 10: Uncertainties of the particle-size determination from the TiRe-LII signal traces due to variations in the underlying heat-up temperature (a), bath-gas temperature (b), and aggregate size (c) compared to Tab. 1 conditions.

For this analysis, a control LII signal for Tab. 1 conditions is generated. Subsequently, this simulated signal trace is fitted by the LII model by systematically changing the assumed values for the relevant input parameters within a given range (one by one). The differences between the evaluated sizes and the initial particle size for the control signal shows the sensitivity of the particle-size determination on the respective parameters.

\subsection{Particle sizing}

Particle sizes were determined from the LIl-signal traces acquired at $425 \pm 15 \mathrm{~nm}$. The TERF method introduced in section 2.3 was applied for each signal trace. Additionally the monodisperse equivalent mean particle sizes were evaluated by full signal fitting. To investigate the influence of the delay time with the TERF method, it was swept from 20 to 800 ns in 20 ns increments and at each delay $d_{p, s m a l l}$ and 
$d_{\mathrm{p} \text {,large }}$ were calculated (Fig. 11). Similar to the model-based analysis results, $d_{\mathrm{p}, \text { small }}$ and $d_{\mathrm{p} \text {,large }}$ increases with increasing delay time due to the increasing contribution of larger particles in the respective input signal segments.
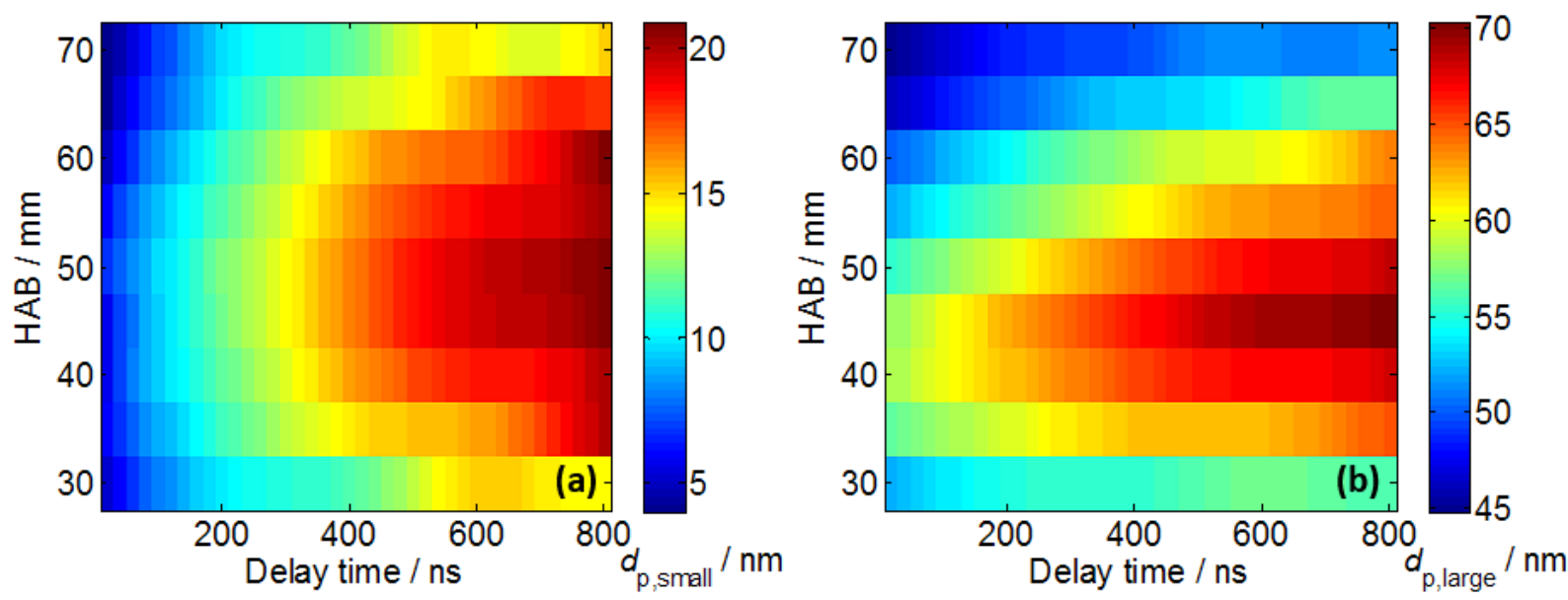

Fig. 11: (a) $d_{\mathrm{p}, \text { small }}$ and (b) $d_{\mathrm{p}, \text { large }}$ at different delay times evaluated for nine HAB positions.

As described in section 2.3, the optimum delay time for each signal depends on the actual particle-size distribution. In an ideal case the evaluated particle size should be least sensitive to changing delay time at around its optimum as it ensures that information blending from different particle size classes is minimal. To define these optimum points, a derivative of the particle sizes (large mode) at each HAB was taken with respect to the changing delay time as shown in Fig. 12. The strong oscillations in the derivative results (for fixed $H A B$ ) were filtered out with a median filter. The optimum delay time is defined as the point where $\Delta d_{\mathrm{p}} / \Delta t$ first approaches $0.1 \mathrm{~nm} / \mathrm{ns}$ and is marked with a white dashed line (parabola). The optimum delay time is as low as $240 \mathrm{~ns}$ at zones where particles are relatively small and reaches values above $600 \mathrm{~ns}$ at flame locations where the largest particle diameters are expected. Any increase in $\Delta d_{\mathrm{p}} / \Delta t$ after this optimum delay can be attributed to noise in the acquired data. 


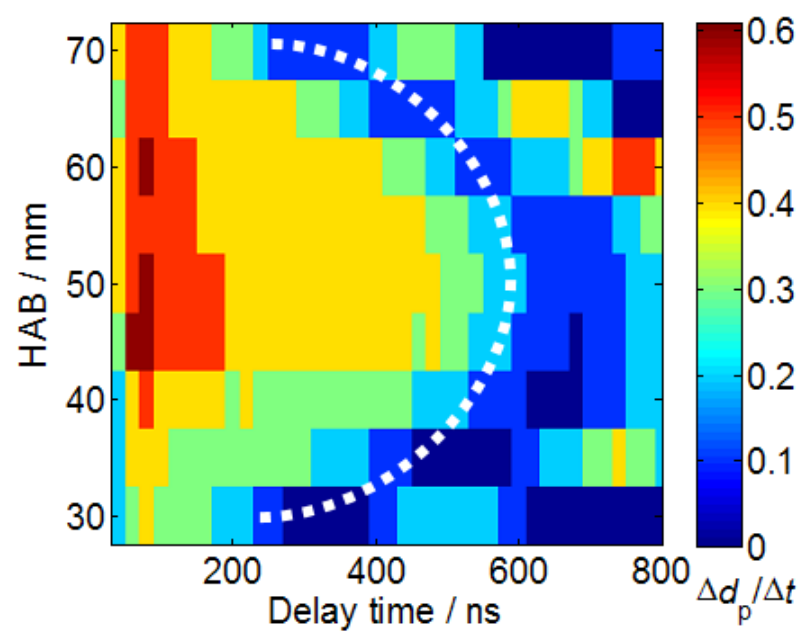

Fig. 12: Derivative of the particle sizes at each HAB with respect to changing delay time.

By using these optimum delay times, $\mathcal{R}_{n}$ at each HAB is calculated and shown in Fig. 13 with the respective $d_{\mathrm{p}, \text { small }}$ and $d_{\mathrm{p} \text {,large }}$. The monodisperse equivalent particle size $d_{\mathrm{p} \text {,mono }}$ is also shown. For all size classes, the particle diameter increases with the flame height and reaches a maximum at $50 \mathrm{~mm} H A B$ for the small mode, and at $45 \mathrm{~mm} \mathrm{HAB}$ for the large mode and the monodisperse equivalent size. After these peak points, particle diameters gradually decrease indicating that soot particle sizes are affected by increasing oxidation. Within the flame height domain between 30 and $70 \mathrm{~mm} \mathrm{HAB}$, the deviation in particle size is $\sim 10 \mathrm{~nm}$ for the small mode and the monodisperse equivalent mean size, whereas it is more than $20 \mathrm{~nm}$ for the large mode.

Fig. 13 also shows that $\mathcal{R}_{n}$ has an inverse relation with particle sizes. In soot formation and oxidation zones the population of small particles with respect to larger ones is greater and reaches its minimum within the zone where the largest particle diameters are evaluated (soot oxidation and soot formation are in balance). This trend of $\mathcal{R}_{n}$ is directly related to different rates of changes in particle size classes. The relative difference of $d_{\mathrm{p} \text {,small }}$ and $d_{\mathrm{p} \text {,large }}$ with respect to $d_{\mathrm{p} \text {,mono }}$ at each flame height shows that the small particles are generally much smaller than the mean particle size in the formation and oxidation zones. This gap reaches its minimum around in the zone where formation and oxidation is balanced whereas the difference between large particles and mean particle size reach its maximum in the zone. 


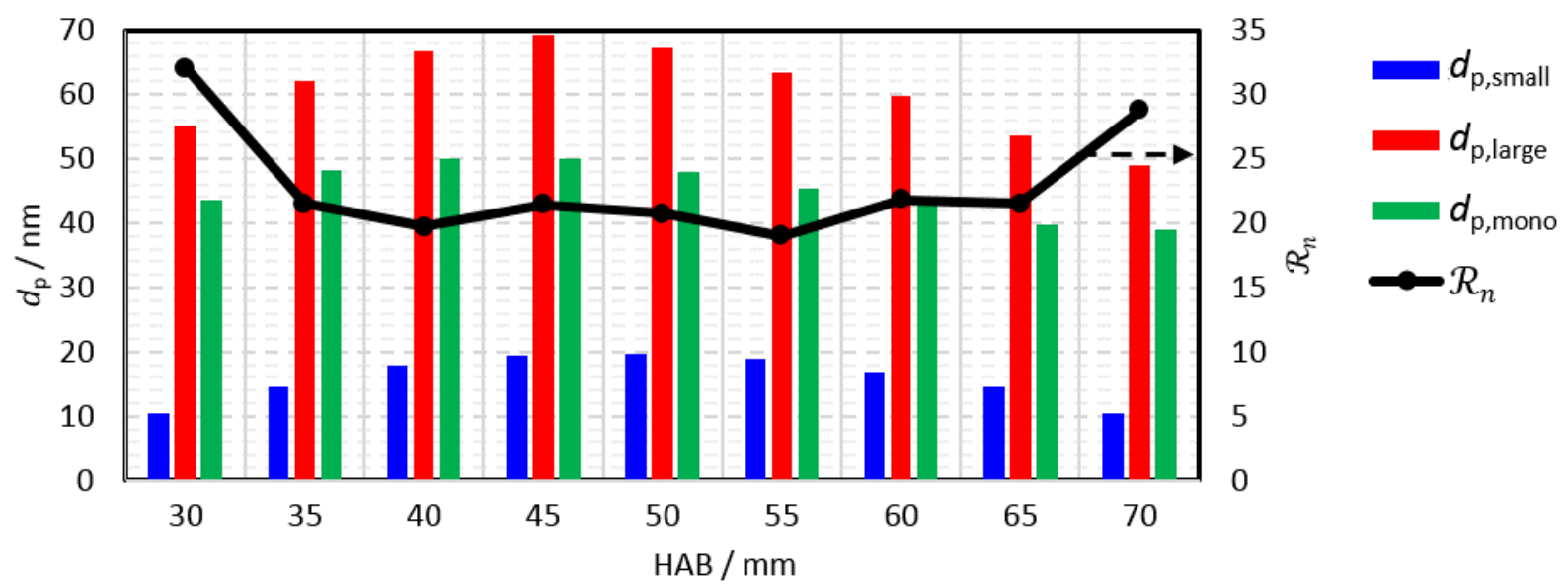

Fig. 13: $d_{p, \text { small }}, d_{p, \text { large }}$ and relative ratio of number densities calculated with the respective optimum delays (cf. Fig. 12) as a function of HAB. $d_{p, \text { mono }}$ is evaluated with full curve-fitting.

\subsection{Comparison of LII and TEM particle-sizing}

The soot-particle size was additionally determined from TEM micrographs acquired at five HAB ranging from 30-70 mm. For each position the obtained primary particle diameters were divided into size bins and represented as a histogram. The histograms were then fitted by lognormal particle-size distributions to obtain continuous distribution functions. A lognormal distribution fits the particle sizes well for all locations (Fig. 14a). The total number of samples $N_{\text {TEM }}$ counted as a function of HAB is given in each graph. The $d_{\mathrm{p}, \text { small }}$ and $d_{\mathrm{p} \text {,large }}$ values obtained from the TERF-derived LII signal analysis for the same locations are also plotted as blue and red dashed vertical lines, resp. $d_{p, \text { mono }}$ values obtained by full curve fitting are shown with green dash-dotted vertical lines. The TEM measurements yielded a similar trend of particle size change as the LII measurements along the flame height. The mean particle size reaches a maximum at $40 \mathrm{~mm} \mathrm{HAB}$ and then gradually decreases due to oxidation.

In most of the locations, $d_{p, \text { large }}$ obtained by LII falls within the particle-size distribution derived from TEM measurements, particularly within the large particle classes. On the other hand, $d_{\mathrm{p}, \text { small }}$ is smaller than the smallest particles measured from TEM for all cases. The main reason for this difference is attributed to the strong bias in evaluating TEM images. Usually, the small particles are either isolated or they exist as small aggregates which reduces the probability of their collision to the lacey carbon film used here (cf. Fig. 14b). Furthermore, as mentioned in section 3, the TEM operator was prone to capturing large aggregates as they provide more information for the statistical analysis of primary particle size measurements (on each grid 40 images were taken) and mostly ignored isolated particles. 
Structures of soot aggregates can also be problematic for the measurement of small particles. Identifying the smaller particles at the center of an aggregate is hindered due to the relatively larger optical density of large particles that cover the small ones. Additionally, even with a small degree of bridging [27] between small and large particles, it becomes more difficult to perceive the spherical structure of small particles and a diameter cannot be measured successfully. Such bridging of small particles was mostly observed at the periphery of the aggregates and two examples are shown in Fig. 14c. These images also indicate that initially loosely connected small individual particles that give rise to rapidly-cooling LII signal might get fused to the larger particles in the supporting agglomerate during sampling and aging in between sampling and TEM measurements. Therefore, the statistical relevance of the TEM-derived number of particles with small diameters may be biased. All these observations indicate that large numbers of very small particles might be systematically overlooked when investigating TEM images of polydisperse soot samples. 

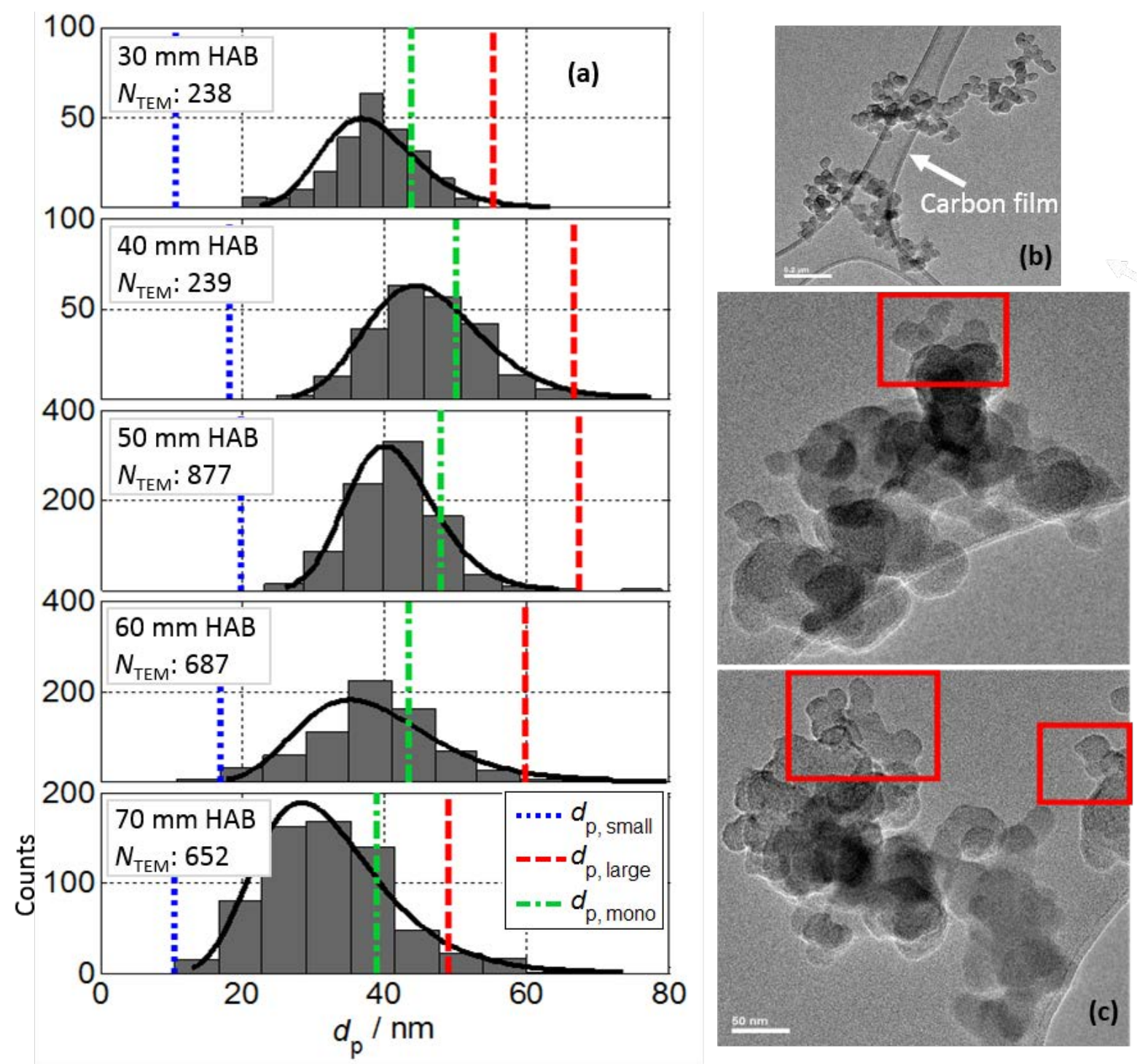

Fig. 14: (a) Classification of primary particle sizes from TEM (gray bars) with lognormal fits (black lines) obtained from soot at five HAB from 30 to $70 \mathrm{~mm}$. LII TERF-derived $d_{\mathrm{p}, \text { small }}$ and $d_{\mathrm{p} \text {, large }}$ values are shown as blue dotted and red dashed vertical lines, resp. $d_{p \text {,mono }}$ values are shown with green dash-dotted vertical lines. (b) Soot aggregates on a lacey carbon film. (c) Bridging of small particles with the larger ones at the aggregate periphery. Due to the lost perception of sphericity, a measurement of diameter is not possible for such particles and therefore they are systematically not included in the statistical analysis. Even smaller particles that might have existed on the agglomerate surface might have got fused entirely with the surface layer and therefore remain invisible.

Another reason for the discrepancy may be the unknown thermal accommodation coefficient, $\alpha_{T}$. Bladh et al. [9] reported that $\alpha_{T}$ depends on the soot morphology and also may decrease with increasing soot maturity (i.e., higher up in the flame). In this work, however, a constant $\alpha_{T}$ was used for all locations 
and all size groups. If a relatively larger $\alpha_{T}$ was used for the younger particles, i.e., smaller particles in the formation zone, a more efficient loss of energy to the surrounding atmosphere would be simulated for these particles. This would eventually lead to a faster signal decay, hence evaluation of relatively larger diameters in the small size classes.

To better understand the discrepancy between the TERF-LII-derived and the TEM-derived small particle sizes, for each HAB a phantom polydisperse TiRe-LIl signal was simulated with the respective TEM distributions shown in Fig. 14a and input parameters shown in Tab. 3. This means that the resulting phantom signal includes no information for particles smaller than what observed in the TEM. Consecutively, $d_{\mathrm{p}, \text { small }}$ and $d_{\mathrm{p} \text {, large }}$ were evaluated with the TERF method. At each HAB, the evaluated $d_{p, s m a l l}$ were found to be larger than the values obtained from the experimental data, cf. Fig. 15. This indicates that the discrepancy between in situ and ex situ measurements is most likely due to the incomplete information derived from TEM.

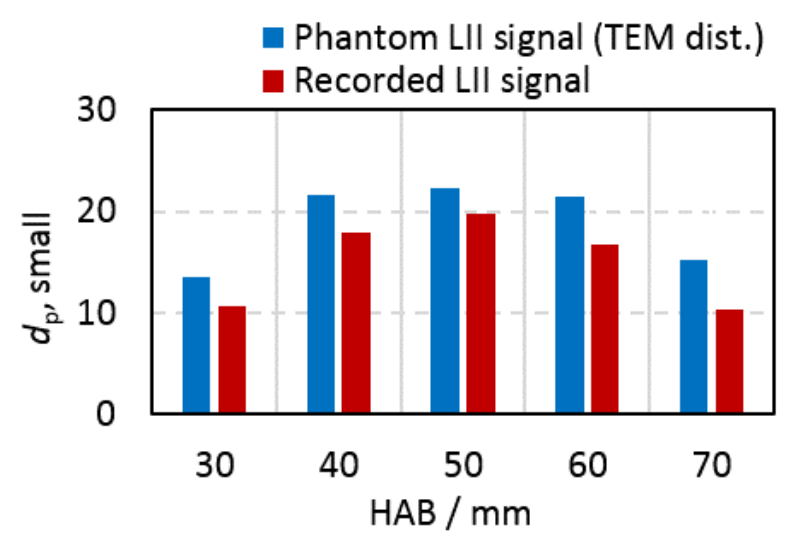

Fig. 15: $d_{\mathrm{p}, \text { small }}$ calculated with acquired LII signal and phantom polydisperse TiRe-LII signal simulated with the respective TEM distributions. The larger $d_{p, s m a l l}$ evaluated with the phantom signal at each HAB indicates that the TEM may fail to provide correct information about the smallest particles that contribute to the LII signal.

For thermophoretic sampling, various exposure times of the grid varying from 25 to $300 \mathrm{~ms}$ were used in the past $[9,23,28]$. In this study, the effect of varying the exposure time on the TEM particle-sizing was investigated by exposing the grid to the flame for 40,60, and $80 \mathrm{~ms}$. The respective particle-size histograms together with their lognormal fit are compared in Fig. 16. The corresponding $d_{\mathrm{cmd}}$ and $\sigma_{\mathrm{g}}$ values are given in each plot. A systematic decrease in the $d_{\mathrm{cmd}}$ and a widening of the distribution is observed with increasing exposure time. The measurements were taken at $50 \mathrm{~mm}$ HAB where soot oxidation dominates and deposited soot particles are exposed to excess oxygen and high temperature. Because the soot particles are only loosely attached to the thin lacey carbon coating, cooling from the 
supporting copper grid is not efficient. During this phase, particles eventually partially oxidize and it cannot be excluded that small particles disappear completely. At the same time, additional particles are continuously deposited until the end of exposure time. Therefore, the width of the particle size distribution $\sigma_{\mathrm{g}}$ increases with increasing exposure time and the distribution increasingly deviates from lognormal. This exposure-time dependent particle-sizing result is in contrast to the conclusion drawn in ref. [23]. Nevertheless, the sampling location in that work is not mentioned which can be the reason of the discrepancy. In fuel-rich zones, deposited particles would not oxidize. Instead, the deposited particles would be subjected to co-aggregation with the latter particles. To minimize the effects of sampling on particle-sizing, the shortest exposure time should be selected that permits collecting sufficient particles for the TEM imaging.

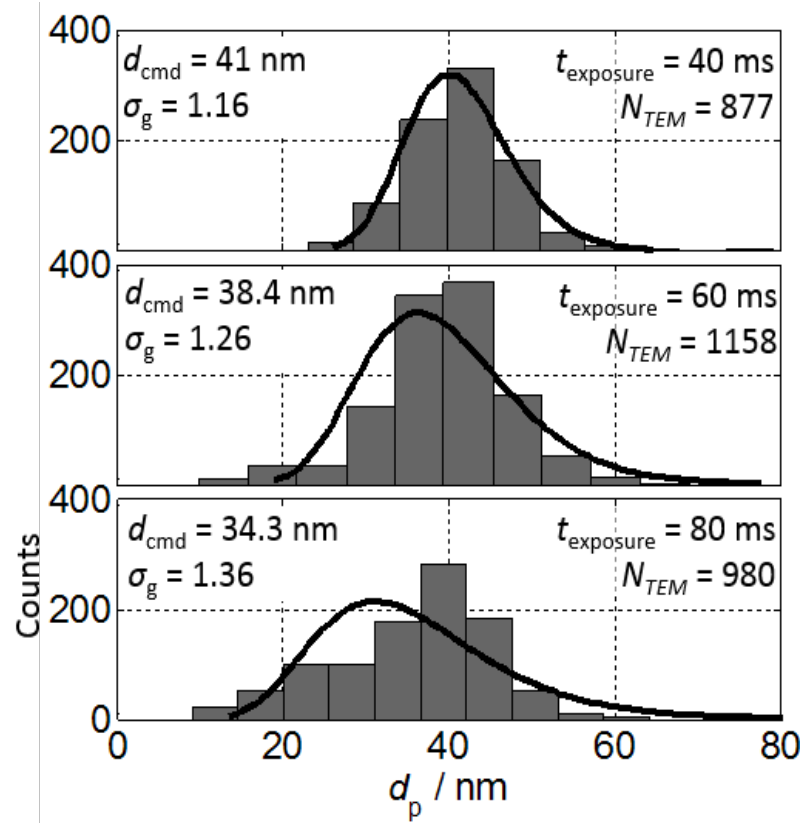

Fig. 16: Histograms of TEM-derived particle sizes with lognormal fits for soot sampled at $50 \mathrm{~mm}$ HAB with varying exposure times.

Perturbations of the flame during sampling can cause additional uncertainty in TEM particle-sizing. The high-repetition-rate movies showed that the flow induced by the moving probe severely distorts the flame. This distortion starts shortly after the probe reaches its stationary position and lasts up to $10 \mathrm{~ms}$. The forced flow and the subsequent unsteadiness of the flame may cause deposition of soot on the grid that stems from locations outside the intended probe volume. 


\section{Conclusions}

A signal-processing method, TERF, was developed for TiRe-LII in polydisperse soot that provides information about the size range and the relative weight of the small and the large fraction of the particle ensemble. The method separates the signal contribution of small and large particles from the overall signal by approximating the LII signal from size classes with narrow size distributions with monoexponential decays. A monodisperse equivalent mean particle size is then evaluated for both size classes and the relative ratio of the number densities of both groups is determined. Compared to timeconsuming polydisperse fitting algorithms, the extracted information is limited. Nevertheless, compared to previous methods it is not necessary to assume the shape of the distribution and the much faster evaluation makes TERF suitable for real-time analysis.

The validity of approximating LII signal traces with mono-exponential decays was analyzed with LII-signal traces simulated for monodisperse soot for various input parameters such as pressure, aggregate size, and heat-up temperature. Suitable ranges of conditions where the TERF method works reliably were determined and the error imposed by the approximation was found to be less than $2 \%$. High heat-up temperatures that cause strong soot evaporation was found to be the conditions where the method cannot be used. The accuracy of the method was tested on simulated signals with lognormal and bimodal particle-size distributions with various distribution parameters. In all cases the method yielded satisfying results.

The TERF method was applied to measured signal traces acquired with a time-resolved detection setup at nine axial locations in a non-premixed atmospheric laminar ethylene/air flame from a Santoro burner. The LII measurements were supported by two-color pyrometry of particle heat-up temperatures. Lowfluence excitation prevented soot evaporation. A model-based analysis was performed to identify the dependence of LII particle-sizing quantitatively on the assumed input parameters such as bath-gas temperature, heat-up temperature, and soot morphology. At each location a monodisperse equivalent mean particle size was also evaluated and compared to TERF method results.

For ex situ characterization, soot particles were sampled at multiple flame heights using a pneumatically-driven soot sampling-probe. Particle size distributions were derived from TEM measurements and compared to the LII-derived results. It was observed that the TERF method provides sizes for the large particle class that are in good agreement with the TEM measurements. Substantial discrepancies were observed, however, between LII and TEM results for the small mode. The 
discrepancies were mainly associated to the biased sampling and TEM operations that omits the analysis of small size classes. The effect of varying exposure times of the TEM grid to the flame was analyzed. At a location with excess oxygen, long exposure times cause an increased oxidation of initially deposited soot, thus the measured mean particle size decreases and the distribution widens. 


\section{References}

[1] L.A. Melton, Appl. Opt. 23 (1984) 2201.

[2] C. Schulz, B.F. Kock, M. Hofmann, H.A. Michelsen, S. Will, B. Bougie, R. Suntz, G.J. Smallwood, Appl. Phys. B 83 (2006) 333.

[3] H.A. Michelsen, F. Liu, B.F. Kock, H. Bladh, A. Boiarciuc, M. Charwath, T. Dreier, R. Hadef, M. Hofmann, J. Reimann, S. Will, P.-E. Bengtsson, H. Bockhorn, F. Foucher, K.-P. Geigle, C. MounaïmRousselle, C. Schulz, R. Stirn, B. Tribalet, R. Suntz, Appl. Phys. B 87 (2007) 503.

[4] M. Hofmann, B.F. Kock, C. Schulz, in:, Eur. Combust. Meet., Chania (Greece), 2007.

[5] M. Charwath, R. Suntz, H. Bockhorn, Appl. Phys. B 104 (2011) 427.

[6] F. Liu, B.J. Stagg, D.R. Snelling, G.J. Smallwood, Int. J. Heat Mass Transf. 49 (2006) 777.

[7] M. Hofmann, B.F. Kock, T. Dreier, H. Jander, C. Schulz, Appl. Phys. B 90 (2007) 629.

[8] B. Menkiel, A. Donkerbroek, R. Uitz, R. Cracknell, L. Ganippa, Combust. Flame 159 (2012) 2985.

[9] H. Bladh, J. Johnsson, N.-E. Olofsson, A. Bohlin, P.-E. Bengtsson, Proc. Combust. Inst. 33 (2011) 641.

[10] S. Banerjee, B. Menkiel, L.C. Ganippa, Appl. Phys. B 96 (2009) 571.

[11] J. Johnsson, H. Bladh, P.-E. Bengtsson, Appl. Phys. B 99 (2010) 817.

[12] A.D. Abid, N. Heinz, E.D. Tolmachoff, D.J. Phares, C.S. Campbell, H. Wang, Combust. Flame 154 (2008) 775.

[13] S. Dankers, A. Leipertz, Appl. Opt. 43 (2004) 3726.

[14] N.A. Fuchs, Geofis. Pura E Appl. 56 (1963) 185. 
[15] F. Liu, K.J. Daun, D.R. Snelling, G.J. Smallwood, Appl. Phys. B 83 (2006) 355.

[16] K.J. Daun, B.J. Stagg, F. Liu, G.J. Smallwood, D.R. Snelling, Appl. Phys. B 87 (2007) 363.

[17] F. Liu, Smallwood, D.R. Snelling, J. Quant. Spectrosc. Radiat. Transf. 93 (2005) 301.

[18] M. Hofmann, Laser-Induced Incandescence for Soot Diagnostics at High Pressure, PhD thesis, Heidelberg University, 2006.

[19] D.R. Snelling, G.J. Smallwood, F. Liu, Ö.L. Gülder, W.D. Bachalo, Appl. Opt. 44 (2005) 6773.

[20] S. Will, S. Schraml, K. Bader, A. Leipertz, Appl. Opt. 37 (1998) 5647.

[21] E. Cenker, G. Bruneaux, L.M. Pickett, C. Schulz, SAE Int. J. Engines 6 (2013) 352.

[22] R.J. Santoro, H.G. Semerjian, R.A. Dobbins, Combust. Flame 51 (1983) 203.

[23] R.A. Dobbins, C.M. Megaridis, Langmuir (1987) 254.

[24] P.B. Kuhn, B. Ma, B.C. Connelly, M.D. Smooke, M.B. Long, Proc. Combust. Inst. 33 (2011) 743.

[25] F. Liu, D.R. Snelling, K.A. Thomson, G.J. Smallwood, Appl. Phys. B 96 (2009) 623.

[26] R.J. Santoro, T.T. Yeh, J.J. Horvath, H.G. Semerjian, Combust. Sci. Technol. 53 (1987) 89.

[27] J. Johnsson, H. Bladh, N.-E. Olofsson, P.-E. Bengtsson, Appl. Phys. B 112 (2013) 321.

[28] K. Tian, K.A. Thomson, F. Liu, D.R. Snelling, G.J. Smallwood, D. Wang, Combust. Flame 144 (2006) 782. 


\section{List of tables}

Tab. 1: Input parameters for modeling the standard "control signal".

Tab. 2: Parameters of the investigated particle-size distributions.

Tab. 3: Heat-up temperature, bath-gas temperature, and aggregate size input parameters imposed for particlesizing at different heights above burner (HAB). 


\section{List of figures}

Fig. 1: Monodisperse particle sizes evaluated from synthetic LII signals simulated for lognormal particle-size distributions with various values of $d_{c m d}$ and $\sigma g$.

Fig. 2: (a) TiRe-LIl signals simulated for soot ensembles with the particle size distributions shown in (b).

Fig. 3: Simulated LII signal for a monodisperse aggregate (conditions given in Tab. 1) and a mono-exponential fit.

Fig. 4: Systematic errors in particle-sizing due to approximating a simulated LII decay with a mono-exponential decay for varying pressure (a), particle size (b), aggregate size (c), and heat-up temperature (d).

Fig. 5: (a) Simulated LII signal for polydisperse soot with a lognormal distribution ( $d_{c m d}=20 \mathrm{~nm}, \sigma g=1.8$, black solid line). A mono-exponential decay is fitted to the section of the LII signal from $300 \mathrm{~ns}$ down to $5 \%$ of the peak signal and extrapolated back into the time domain from 0 to $300 \mathrm{~ns}$ (red dashed line). The difference between both curves represents the signal contribution from small particles (blue dotted line). (b) The same data normalized to the respective peak values.

Fig. 6: Lognormal distribution function of particle sizes for $d_{c m d}=20 \mathrm{~nm}$ and $\sigma g=1$.8. The vertical lines show the monodisperse equivalent particle sizes evaluated from the exponential decays (see text) representing the small and large particle class of $d_{p, \text { small }}=15 \mathrm{~nm}$ and $d_{p, \text { large }}=57 \mathrm{~nm}$, respectively.

Fig. 7: (a) $d_{p, \text { small }}$ and $d_{p, \text { large }}$ and (b) ratio of the number densities as a function of the chosen delay time.

Fig. 8: Probability density functions for lognormal $(a)$ and bimodal $(b, c)$ distributions. Vertical lines show the monodisperse equivalent particle sizes evaluated for the small and large particle classes.

Fig. 9: Experimental arrangement

Fig. 10: Uncertainties of the particle-size determination from the TiRe-LII signal traces due to variations in the underlying heat-up temperature (a), bath-gas temperature (b), and aggregate size (c) compared to Tab. 1 conditions.

Fig. 11: (a) $d_{p, \text { small }}$ and (b) $d_{p, \text { large }}$ at different delay times evaluated for nine HAB positions.

Fig. 12: Derivative of the particle sizes at each $H A B$ with respect to changing delay time.

Fig. 13: $d_{p, \text { small, }} d_{p, \text { large }}$ and relative ratio of number densities calculated with the respective optimum delays (cf. Fig.

12) as a function of HAB. $d_{p, \text { mono }}$ is evaluated with full curve-fitting.

Fig. 14: (a) Classification of primary particle sizes from TEM (gray bars) with lognormal fits (black lines) obtained from soot at five HAB from 30 to $70 \mathrm{~mm}$. LII TERF-derived $d_{p, \text { small }}$ and $d_{p, \text { large }}$ values are shown as blue dotted and red dashed vertical lines, resp. $d_{p \text {,mono }}$ values are shown with green dash-dotted vertical lines. (b) Soot aggregates on a lacey carbon film. (c) Bridging of small particles with the larger ones at the aggregate periphery. Due to the lost perception of sphericity, a measurement of diameter is not possible for such particles and therefore they are systematically not included in the statistical analysis. Even smaller particles that might have existed on the agglomerate surface might have got fused entirely with the surface layer and therefore remain invisible. 
Fig. 15: $d_{p, \text { small }}$ calculated with acquired LII signal and phantom polydisperse TiRe-LII signal simulated with the respective TEM distributions. The larger $d_{p, \text { small }}$ evaluated with the phantom signal at each HAB indicates that the TEM may fail to provide correct information about the smallest particles that contribute to the LII signal.

Fig. 16: Histograms of TEM-derived particle sizes with lognormal fits for soot sampled at 50 mm HAB with varying exposure times. 